\title{
Effect of rosiglitazone on 20-hydroxyeicosatetraenoic acid levels and CYP4F2 expression in HepG2 cells
}

\author{
Yazun Jarrar ${ }^{1,2}$, Su-Jun Lee ${ }^{1 *}$ \\ ${ }^{1}$ Department of Pharmacology and Pharmacogenomics Research Center, Inje University College of Medicine, Inje University, \\ Busan, South Korea, ${ }^{2}$ Department of Pharmacy, College of Pharmacy, Alzaytoonah University of Jordan, Amman, Jordan \\ *For correspondence: Email: 2sujun@inje.ac.kr; Tel: +82 (51) 890-5911
}

\begin{abstract}
Purpose: To determine the effect of rosiglitazone on the levels of the cardiotoxic arachidonic acid metabolite, 20-hydroxyeicosatetraenoic acid (20-HETE), in the human liver hepatocellular carcinoma cell line, HepG2.

Methods: HepG2 cells were treated with thiazolidinedione rosiglitazone and the mRNA and protein expressions of cytochrome P450 4F2 (CYP4F2) responsible for synthesizing 20-HETE were measured using quantitative real-time polymerase chain reaction (qRT-PCR) and western blotting. The levels of 20-HETE were evaluated using liquid chromatography/mass spectrometry (LC-MS).

Results: Rosiglitazone significantly increased the levels of CYP4F2 mRNA and protein when compared with the control group $(p<0.05)$. This was correlated with significantly increased 20-HETE levels in the culture medium of rosiglitazone-treated cells in a dose-dependent manner $(p<0.05)$. The PPARY antagonist, GW9662, significantly repressed the increased production of 20-HETE and CYP4F2 mRNA protein $(p<0.05)$.

Conclusion: Rosiglitazone increases the synthesis of 20-HETE via activation of PPARY receptor and upregulation of CYP4F2. These findings may provide an additional explanation, at least in part, for the unwanted side effects of rosiglitazone on the cardiovascular system.
\end{abstract}

Keywords: Thiazolidinediones; 20-HETE, CYP4, PPARY, HepG2 cells

\begin{abstract}
This is an Open Access article that uses a fund-ing model which does not charge readers or their institutions for access and distributed under the terms of the Creative Commons Attribution License (http://creativecommons.org/licenses/by/4.0) and the Budapest Open Access Initiative (http://www.budapestopenaccessinitiative.org/read), which permit unrestricted use, distribution, and reproduction in any medium, provided the original work is properly credited.
\end{abstract}

Tropical Journal of Pharmaceutical Research is indexed by Science Citation Index (SciSearch), Scopus, International Pharmaceutical Abstract, Chemical Abstracts, Embase, Index Copernicus, EBSCO, African Index Medicus, JournalSeek, Journal Citation Reports/Science Edition, Directory of Open Access Journals (DOAJ), African Journal Online, Bioline International, Open-J-Gate and Pharmacy Abstracts

\section{INTRODUCTION}

Thiazolidinediones are activators of peroxisome proliferator-activated receptor gamma (PPARY). These compounds decrease insulin resistance and are used in the treatment of diabetes mellitus type II [1]. Two thiazolidinediones are currently available for clinical use: pioglitazone and rosiglitazone [2]. The adverse effect most commonly caused by thiazolidinediones are weight gain and cardiotoxicity [1]. Thiazolidinediones can cause myocardial infarction and increase cardiac-related mortality [3]. Rosiglitazone was evaluated for cardiac outcomes in a clinical trial study and it found out that hospitalization, due to cardiovascular event, was more significant in diabetic patients receiving rosiglitazone, in combination with other anti-diabetic drugs [4]. In addition, it was in a study of the United Kingdom Prospective 
Diabetes group, that combination of rosiglitazone with metformin and sulfonylurea increased diabetes-related mortality [5]. However, the detailed mechanism of rosiglitazone-induced cardiotoxicity remains unclear.

20-Hydroxyeicosatetraenoic acid (20-HETE) is a metabolite of arachidonic acid that synthesized by cytochrome P450 (CYP450) enzymes, mainly CYP4 subfamily members including CYP4A11, CYP4F2, CYP4F3, and CYP4V2 [6]. 20Hydroxyeicosatetraenoic acid is produced in many organs, including the liver [7]. 20Hydroxyeicosatetraenoic acid affects the cardiovascular system as it increases the contractility of blood vessels and platelet aggregation [8]. High 20-HETE levels increase the occurrence of chronic diseases, especially cardiovascular diseases [9]. It was found that 20HETE stimulates the renin-angiotensinaldosterone pathway in the kidney that results in increasing blood pressure and the risk of other cardiovascular diseases, such heart failure [10]. 20-Hydroxyeicosatetraenoic acid levels are also increased in rofecoxib- [9] and doxorubicininduced cardiotoxicity [11]. Furthermore, the expression of 20-HETE-synthesizing CYP450s was significantly upregulated in the mouse heart after non-steroidal anti-inflammatory drug (NSAID) treatment, which was correlated with toxicological changes in the heart [12].

Although 20-HETE is involved, at least partly, in drug-induced cardiotoxicity [12], no study has investigated the effect of cardiotoxic rosiglitazone on 20-HETE production. Peroxisome proliferatoractivated receptor nuclear receptors regulate the expression of CYP4 family genes. Yi et al found that PPARy controls the expression of CYP4V2 in macrophages [13]. Therefore, this study hypothesized that rosiglitazone affects 20-HETE production via a PPAR-dependent mechanism. Accordingly, the current study investigated the effect of rosiglitazone on the expression of 20HETE-synthesizing CYP4 genes and the production of 20-HETE in HepG2 cells.

\section{EXPERIMENTAL}

\section{Chemicals and reagents}

Rosiglitazone and a PPARy antagonist (GW9662) were purchased from Sigma Aldrich (St. Louis, MO, USA). TRIzol, DNase, 10x TaqMan, and Moloney murine leukemia virus (MMLV) reverse transcriptase were obtained from Invitrogen (Carlsbad, CA, USA). 20Hydroxyeicosatetraenoic acid and 20-HETE-d6 were purchased from Cayman (Ann Arbor, MI, USA). Antibodies and chemiluminescence kits for
CYP4F2 and glyceraldehyde phosphate dehydrogenase (GAPDH) were purchased from Santa Cruz Biotechnology (Santa Cruz, CA, USA).

\section{Cell culture}

Human HepG2 liver cell line was obtained from the American Type Culture Collection (Rockville, MD, USA). The HepG2 cells were incubated at $37{ }^{\circ} \mathrm{C}$ with $5 \% \mathrm{CO}_{2}$ in $1 \%$ penicillin and streptomycin in RPMI 1640 medium containing $10 \%$ fetal bovine serum. The HepG2 cells in sixwell plates were treated with $1 \%$ DMSO (control group), or with 1 and $10 \mu \mathrm{M}$ of rosiglitazone. The cells were incubated with cell media containing rosiglitazone for $24 \mathrm{~h}$. To determine the role of PPARy receptors in CYP4 expression and 20HETE production, the PPARY antagonist GW9662 (10 $\mu \mathrm{M})$ was added $3 \mathrm{~h}$ before thiazolidinedione treatment.

\section{Assay of mRNA expression}

RNA was isolated from HepG2 cells using TRIzol reagent. The extracted RNA was incubated with 1 unit of DNase to remove genomic DNA. Then, cDNA was synthesized by M-MLV reverse transcriptase enzyme at $42{ }^{\circ} \mathrm{C}$ for 1 hour. Quantitative real time PCR probes for the CYP4F2 and GAPDH genes were obtained from Applied Biosystems (Foster City, CA, USA). The real-time PCR condition was as followings: Initial $95^{\circ} \mathrm{C}$ for 10 minutes, followed by 40 cycles of 95 ${ }^{\circ} \mathrm{C}$ for 20 seconds and $60{ }^{\circ} \mathrm{C}$ for 1 minute. The expressions of the target genes were normalized to that of the GAPDH gene using the formula $2^{\triangle \Delta C T}$.

\section{Immunoblot assay}

Western blot analysis of CYP4F2 protein was done as described previously [7]. HepG2 cells were lysed and the proteins were extracted using RIPA buffer contained $150 \mathrm{mM} \mathrm{NaCl}, 1 \%$ sodium deoxycholate, $2.5 \mathrm{mM}$ sodium pyrophosphate, and $1 \mathrm{mM} \beta$-glycerophosphate. The extracted proteins were electrophoresed at $80 \mathrm{~V}$ on $10 \%$ polyacrylamide gels, and transferred to polyvinylidene difluoride membranes and electrophoresed at $85 \mathrm{~V}$ for 60 $\min$. Then, the membranes were blocked with 5 $\%$ skim milk solution for $2 \mathrm{~h}$, followed by incubation with primary CYP4F2 antibodies for 1 h. Then, the membranes were washed and incubated with secondary horseradish peroxidase-conjugated anti-lgG antibodies overnight at $4{ }^{\circ} \mathrm{C}$. The CYP4F2 and GAPDH proteins were detected using the ECL method (GE Healthcare Bio-Sciences, Little Chalfont, 
Buckinghamshire, UK). The density of the bands was quantified using a MultiGauge (Fuji Photo Film, Science Laboratory, Tokyo, Japan). The relative CYP4F2 protein levels were normalized to the control GAPDH protein.

\section{Quantification of 20-HETE}

The amount of 20-HETE produced in the cell medium was measured using liquid chromatography-mass spectrometry with an API 3000 LC-MS/MS system (Agilent Technologies, Santa Clara, CA, USA), as described previously [13]. The chemical compounds, in the cell media of HepG2 cells, were separated on a reversephase column Atlantis dC18 (Waters, Ireland). The mobile solvent consisted of $0.1 \%$ formic acid, acetonitrile, and water. The mass transitions for detecting of 20-HETE and 20HETE-d6 were 319-301 and 281-3, respectively. 20-Hydroxyeicosatetraenoic acid -d6 (100 $\mathrm{pg} / \mathrm{mL}$ ) was used as the internal standard.

\section{Statistical analysis}

All results are presented as mean \pm standard deviation. Analysis of variance (ANOVA) followed by Newman-Keuls post-hoc test was used as a statistical tool for analyzing the difference between the tested groups. The $p$-value was considered significant at $<0.05$. All statistical analyses were performed using SAS (ver. 9.1.3; SAS Institute, Cary, NC, USA).

\section{RESULTS}

\section{Effect of rosiglitazone on CYP4F2 mRNA expression}

Quantitative RT-PCR analysis of the CYP4F2 gene showed a concentration-dependent increase in the expression of CYP4F2 in HepG2 cells treated with rosiglitazone (Figure 1). CYP4F2 mRNA expression was increased approximately two-fold with $10 \mu \mathrm{M}$ rosiglitazone compared with DMSO $(p<0.05)$. When the cells were pretreated with GW9662, CYP4F2 mRNA expression was significantly $(p<0.05)$ lower than in HepG2 cells treated with $10 \mu \mathrm{M}$ rosiglitazone only.

\section{Effect of rosiglitazone on CYP4F2 protein expression}

Rosiglitazone significantly increased ( $p<0.05$ ) CYP4F2 protein expression in a dose-dependent manner and pretreating the cells with GW9662 significantly attenuated this increase (Figure 2).

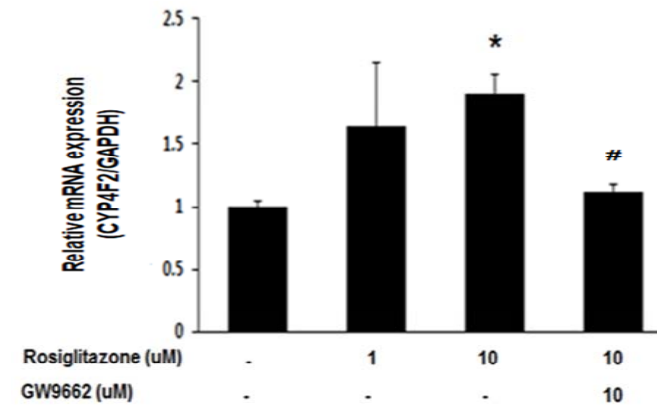

Figure 1: Expression of CYP4F2 mRNA in HepG2 cells following rosiglitazone treatment. Data are representative of three independent experiments. ${ }^{*} p<$ 0.05 , indicates a significant difference compared with the DMSO vehicle group; $\# p<0.05$, indicates a significant difference compared with the rosiglitazone $(10 \mu \mathrm{M})$-treated group

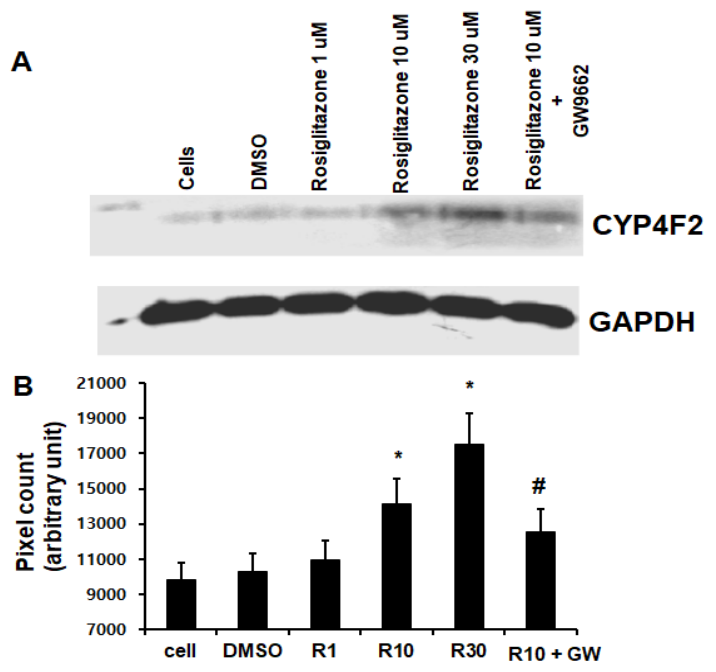

Figure 2: Western blot analysis of CYP4F2 protein in HepG2 cells with rosiglitazone treatment. Data are representative of three independent experiments. (A) CYP4F2 immunoreactive proteins in cellular fractions from HepG2 cells treated with rosiglitazone and the PPAR-y antagonist Gw9662. (B) Relative pixel values of the CYP4F2 protein were normalized to GAPDH levels. ${ }^{*} p<0.05$, indicates a significant difference compared with the DMSO vehicle group; $\# p<0.05$, indicates a significant difference compared with the rosiglitazone $(10 \mu \mathrm{M})$-treated group. $\mathrm{R} 10$ is the abbreviation of $10 \mu \mathrm{M}$ rosiglitazone, R30 is the abbreviation of $30 \mu \mathrm{M}$ rosiglitazone and $\mathrm{GW}$ is the abbreviation of PPARy antagonist GW9662

\section{Effects of rosiglitazone on 20-HETE production in HepG2 cells}

Figure 3 shows 20-HETE levels in HepG2 cell medium with different thiazolidinedione treatments. Rosiglitazone increased 20-HETE levels in the cell medium in a concentrationdependent manner. 20-Hydroxyeicosatetraenoic 
acid levels were decreased significantly ( $p<$ 0.05 ) when the cells were pretreated with GW9662.

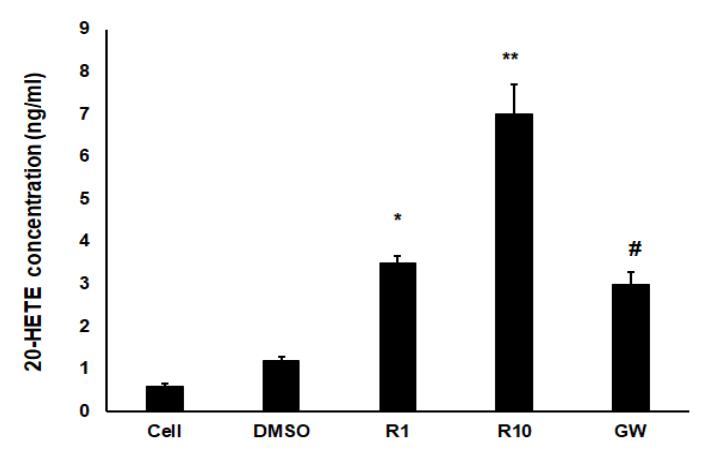

Figure 3: 20-HETE levels in HepG2 cell medium following rosiglitazone treatment. 20-HETE levels were analyzed using liquid chromatography-mass spectrometry. ${ }^{*} p<0.05$, indicates a significant difference compared with the DMSO vehicle group; \#p $<0.05$, indicates a significant difference compared with the rosiglitazone $(10 \mu \mathrm{M})$-treated group. $\mathrm{R} 1$ is the abbreviation of $1 \mu \mathrm{M}$ rosiglitazone, R10 is the abbreviation of $10 \mu \mathrm{M}$ rosiglitazone and $\mathrm{GW}$ is the abbreviation of PPARY antagonist GW9662

\section{DISCUSSION}

20-Hydroxyeicosatetraenoic acid has been found to be associated with increased risk of druginduced cardiotoxicity $[11,12]$. The present study investigated the influence of rosiglitazone, which is known to cause cardiotoxicity, on the production of 20-HETE. It has been found, in this study, that rosiglitazone increased the production of 20-HETE via activation of the PPARy receptor and induced the expression of 20-HETEsynthesizing CYP4F2 enzyme. This may provide an additional explanation for the unwanted side effects of rosiglitazone on the cardiovascular system at least in part.

The current study used HepG2 cells because the liver is a major site of drug metabolism and 20HETE synthesis [14]. In addition, HepG2 cells have been used for studying the upregulation of P450s [15]. The expression of CYP450s was reported to be reduced in liver cell lines compared with primary hepatic cells and tissues freshly isolated from humans or animals [16]. However, it was found that there was a similarity in the pattern of P450 induction in both HepG2 cells and primary hepatocytes [17]. The current study confirmed the expression of 20-HETEsynthesizing CYP4F2 in HepG2 cells using reverse-transcriptase PCR (data not shown). Therefore, HepG2 cells are capable of producing 20-HETE via the expressed CYP4s and could be used as a model cell line to study the effect of thiazolidinediones on 20-HETE production.

The present study also attempted to study the effect of thiazolidinediones on another major 20HETE-synthesizing enzyme, CYP4A11. However, this study was unable to find out CYP4A11 in HepG2 cells (data not shown). Therefore, the present research studied the effect of rosiglitazone on the expression of CYP4F2 and 20-HETE production in HepG2 cells.

The expression of CYP4F2 mRNA was increased significantly after rosiglitazone treatment in a dose-dependent manner in the assay of quantitative real-time PCR. The upregulated expression of 20-HETE-synthesizing enzymes is associated with NSAID-induced cardiotoxicity [12]. Some studies have reported that the mRNA expression of specific genes is not correlated with protein synthesis [18]. Therefore, the present study investigated the effect of rosiglitazone on the expression of CYP4F2 protein and found that it was also significantly induced in a dose-dependent manner.

There are 13 CYP4 isoforms in the human genome and seven are CYP4F proteins [6]. Because of their close structural similarities within the same CYP4F subfamily, there are no specific antibodies for each CYP4F protein. Therefore, although immunoblotting results indicated increased CYP4F2 expression, it cannot be ruled out the possibility that other similar CYP4F proteins could be detected together with the CYP4F2 protein.

Levels of the 20-HETE-synthesizing protein CYP4 have been shown to be increased in doxorubicin-induced cardiotoxicity [11]. Since mRNA and protein levels of 20-HETEsynthesizing CYP4F2 were induced following rosiglitazone treatment, it can be concluded that the increased CYP4F2 levels were due to the upregulated mRNA expression.

It was observed in this study that rosiglitazone increased 20-HETE synthesis in HepG2 cells by 5-fold, while CYP4F2 mRNA was upregulated by 2 folds. This might indicate the involvement of other 20-HETE-synthesizing P450s that were not analyzed in this study.

The PPARy receptor is activated by fatty acids and regulates many genes, including members of the CYP4 subfamily [13]. Since the PPARY receptor antagonist GW9662 significantly inhibited the induction of CYP4F mRNA and 
protein by rosiglitazone treatment, the upregulation of CYP4F2 mRNA and protein expression appeared to be due to activation of the PPARy receptor. Furthermore, insufficient 20-HETE was synthesized in the presence of GW9662, indicating that PPARY activation is essential for CYP4F2 expression. Therefore, the underlying mechanism of rosiglitazone on 20HETE production appears to be a PPARydependent pathway via CYP4 induction. In this study, it was noticed the possible role of the PPARy receptor in thiazolidinedione-induced cardiotoxicity by showing that rosiglitazone increases the production of cardiotoxic 20-HETE through a PPARy-dependent mechanism.

\section{CONCLUSION}

The findings of this study demonstrate that rosiglitazone enhances the production of 20 HETE in HepG2 cells via a PPARy-dependent mechanism and also induces 20-HETEsynthesizing CYP4F2 enzyme. These findings may provide, at least in part, an additional explanation for the unwanted side effects of rosiglitazone on the cardiovascular system.

\section{DECLARATIONS}

\section{Acknowledgement}

This work was supported by 2017 Inje University research grant.

\section{Conflict of interest}

No conflict of interest is associated with this work.

\section{Contribution of authors}

We declare that this work was done by the authors named in this article and all liabilities pertaining to claims relating to the content of this article will be borne by the authors.

\section{Open Access}

This is an Open Access article that uses a funding model which does not charge readers or their institutions for access and distributed under the terms of the Creative Commons Attribution License (http://creativecommons.org/licenses/by/ 4.0) and the Budapest Open Access Initiative (http://www.budapestopenaccessinitiative.org/rea d), which permit unrestricted use, distribution, and reproduction in any medium, provided the original work is properly credited.

\section{REFERENCES}

1. Derosa G, Maffioli P. Peroxisome proliferator-activated receptor-gamma (PPAR-gamma) agonists on glycemic control, lipid profile and cardiovascular risk. Curr Mol Pharmacol 2012; 5: 272-281.

2. Diamant $M$, Heine $R J$. Thiazolidinediones in type 2 diabetes mellitus: current clinical evidence. Drugs 2003; 63: 1373-1405.

3. Ye J. Challenges in Drug Discovery for Thiazolidinedione Substitute. Yao Xue Xue Bao 2011; 1: 137-142.

4. Home PD, Pocock SJ, Beck-Nielsen $H$, Gomis $R$, Hanefeld M, Dargie H, Komajda M, Gubb J, Biswas N, Jones NP. Rosiglitazone Evaluated for Cardiac Outcomes and Regulation of Glycaemia in Diabetes (RECORD): study design and protocol. Diabetologia 2005; 48: 1726-1735.

5. Effect of intensive blood-glucose control with metformin on complications in overweight patients with type 2 diabetes (UKPDS 34). UK Prospective Diabetes Study (UKPDS) Group. Lancet 1998; 352: 854-865.

6. Jarrar YB, Lee SJ. Molecular Functionality of Cytochrome P450 4 (CYP4) Genetic Polymorphisms and Their Clinical Implications. Int J Mol Sci 2019; 20.

7. Jarrar YB, Cho SA, Oh KS, Kim DH, Shin JG, Lee SJ. Identification of cytochrome P450s involved in the metabolism of arachidonic acid in human platelets. Prostaglandins Leukot Essent Fatty Acids 2013; 89: 227-234.

8. Liu JY, Li N, Yang J, Li N, Qiu H, Ai D, Chiamvimonvat N, Zhu Y, Hammock BD. Metabolic profiling of murine plasma reveals an unexpected biomarker in rofecoxibmediated cardiovascular events. Proc Natl Acad Sci U S A 2010; 107: 17017-17022.

9. Rocic P, Schwartzman ML. 20-HETE in the regulation of vascular and cardiac function. Pharmacol Ther 2018; 192: 74-87.

10. Waldman M, Peterson SJ, Arad M, Hochhauser E. The role of 20-HETE in cardiovascular diseases and its risk factors. Prostaglandins Other Lipid Mediat 2016; 125: 108-117.

11. Zordoky BN, Anwar-Mohamed A, Aboutabl ME, El-Kadi AO. Acute doxorubicin cardiotoxicity alters cardiac cytochrome $P 450$ expression and arachidonic acid metabolism in rats. Toxicol Appl Pharmacol 2010; 242: 38-46.

12. Jarrar YB, Jarrar $Q$, Abed A, Abu-Shalhoob M. Effects of nonsteroidal anti-inflammatory drugs on the expression of arachidonic acid-metabolizing Cyp450 genes in mouse hearts, kidneys and livers. Prostaglandins Other Lipid Mediat 2019; 141: 14-21.

13. Yi M, Shin JG, Lee SJ. Expression of CYP4V2 in human THP1 macrophages and its transcriptional regulation by peroxisome proliferator-activated receptor gamma. Toxicol Appl Pharmacol 2017; 330: 100-106.

14. Jarrar YB, Cha EY, Seo KA, Ghim JL, Kim HJ, Kim DH, Lee SJ, Shin JG. Determination of major UDPglucuronosyltransferase enzymes and their genotypes

Trop J Pharm Res, April 2021; 20(4): 707 
responsible for 20-HETE glucuronidation. J Lipid Res 2014; 55:2334-2342.

15. Zanger UM, Schwab M. Cytochrome P450 enzymes in drug metabolism: regulation of gene expression, enzyme activities, and impact of genetic variation. Pharmacol Ther 2013; 138: 103-141.

16. Westerink WM, Schoonen WG. Cytochrome P450 enzyme levels in HepG2 cells and cryopreserved primary human hepatocytes and their induction in HepG2 cells. Toxicol In Vitro 2007; 21: 1581-1591.
17. Berger B, Donzelli M, Maseneni S, Boess F, Roth A, Krahenbuhl S, Haschke M. Comparison of Liver Cell Models Using the Basel Phenotyping Cocktail. Front Pharmacol 2016; 7: 443.

18. Edfors F, Danielsson F, Hallstrom BM, Kall L, Lundberg $E$, Ponten F, Forsstrom B, Uhlen M. Gene-specific correlation of RNA and protein levels in human cells and tissues. Mol Syst Biol 2016; 12: 883. 